\title{
Multi-mode and band Terminal access policy based on power macro I micro base station
}

\author{
HE Qingsu ${ }^{1, a}$, OU Qinghai ${ }^{2, b}$, LI Lili ${ }^{1, c}$, LI Wenjing ${ }^{2, d}$, LIU Zhu ${ }^{2, e}$ \\ ${ }^{1}$ Beijing Huitong Financial Information Technology CO.LTD, Beijing 100053, China \\ ${ }^{2}$ State Grid Information \& Telecommunication Group. CO. LTD, Beijing 100031, China \\ aemail: heqingsu@sgitg.sgcc.com.cn, bemail: ouqing- \\ hai@sgitg.sgcc.com.cn, \\ jing@sgitg.sgcc.com.cn, \\ cemail: lilili20070062@126.com, demail: liwen- \\ eemail: liuzhu@sgitg.sgcc.com.cn
}

\begin{abstract}
Keywords: macro and micro base station pool; TDD / FDD common mode networking technologies; multimode base station; unlimited relay
\end{abstract}

\begin{abstract}
According to the actual application of power wireless communication system, wireless network communication in power system mainly hire telecom operators GPRS /CDMA wireless network, it is evolution from $2 \mathrm{G}$ to $3 \mathrm{G}, 4 \mathrm{G}$, and several networks coexist, the power terminal how to deal with the influence of the wireless network upgrade of the operator, what to do ensure that the power wireless communication business information can be stable transmission in wireless communication network of 2/3/4G multi-mode multi frequency, based on multi-mode multi frequency access technology, this paper presents the technological scheme of multi-mode multi frequency power terminal access network, effectively solve the problem of upgrading the power terminal caused by the operator's wireless network upgrade, saving the cost of wireless network transformation.
\end{abstract}

\section{Introduction}

With the smart grid communication needs to become more diversified, long-term evolution (Long Term Evolution, LTE) technology as the representative of the new private network wireless communication technology in electric power communication system are more and more attention. New wireless communication technology has incomparable advantage in terms of transmission speed, reliability, ease of maintenance and real-time aspects, which is able to accommodate the large number of natural communication terminals, the communication distance is short, a small node traffic data by distribution network urban construction and expansion of high-impact communications access networks with electricity. Each business area of existing power communication network is an independent production and development, therefore with the corresponding communications network has been based on the business need for a separate building, until the State Grid Corporation of "the 12th Five-Year Plan" communications network planning was first proposed the concept of "Electricity terminal communication access ". Previously separate mode of network communication can neither make full use of resources, nor conducive to the unity of equipment operation and maintenance management. Therefore, unified physical network to carry multiple power communications services in the terminal communication access network is the future development direction of network construction access mode. Multi-service bearing building ideas in the telecommunications industry and electricity major communications core network. Data communication network already mature application, but in the research field of electric power communication access network has just launched, has not yet mature research results. Currently, wireless access is the coexistence of multiple standards, and in among of the update and evolution, hence the research of the power terminal access techniques to support multi-mode multi-band wireless communication, which is to achieve seamless integration the 4G public network with Power Communication Network, in order to enhance wireless communications technology in the Technology and Economic Advancement power system application process, also improve the power communication network for mobile Internet and other multi-service bearing capability, 
thereby contributing to power wireless communication access smooth upgrade[1].

\section{Critical technologies of Multi-mode multi-band access}

\section{Macro and micro cell base station.}

The era of 4G network faces four major challenges: (1)strong depth coverage needs; (2)high quality requirements of mobile data services; (3)High frequency loss and poor coverage; (4) New sites difficulty. These four challenges spawned the demand for rich base stations form. According to ABI Research, shipments of LTE base stations will exceed the small LTE macro base station by 2014[2].

Currently, LTE base station products includes macro base stations, micro base stations, pico-base stations and Relay and so on. Among them, the macro and micro base stations can be further divided into two kinds of integration and distributed from the hardware architecture. Micro station can be divided into micro-station integration and distributed micro station (BBU + micro RRU). In the domestic TD-LTE scale test network, LTE base stations has been applied in a variety of forms. In the early days of the network construction, network coverage is the main demand and the macro base station deployed in active form; when the construction of the network reaches a certain size, network capacity become the main demand and the micro base station is the main solution.

Under the electricity industry-specific application scenarios, power terminals access network using the 4G network. In the early deployment of the network, wireless broadband communication network construction purpose is "low-cost, wide coverage" and the main target is achievement of wide coverage. Various types of electrical business communication terminal penetration rate is not high and the network traffic load is not heavy, 4G network macro base station should be widely constructed. After the initial construction of a wide range of communication network, network deployment begin to enter the stage of development. Learn from foreign $4 \mathrm{G}$ network deployment operating experience, communications of a large number of business data will lead to transmission load grow rapidly. In the situation of tense macro cell density and frequency resources, the construction of the network should focus on how to solve the problem of coverage blind spots and weak coverage area. Considering micro base station solutions and development as the main direction and making it an effective complement to the macro base station enhance the capacity of the $4 \mathrm{G}$ network.

\section{TDD / FDD common mode networking technology.}

According to the conditions of current standards and technical, the access side using TDD or FDD format is completely transparent for the core network. Theoretically, core network can be completely shared between LTE TDD and LTE FDD. For the access side, specific technology is shown in Fig1. On the base of original FDD base station, common mode networking technology focused on aspects of radio upgrade, added TDD remote radio unit (RRU) and TD-LTE channel board, TDD and FDD shared signal BBU (antenna feeder, main control board, power supply, the alarm module) 、 facilities (tower, transmission, power supply, etc.) and EPC and so on.

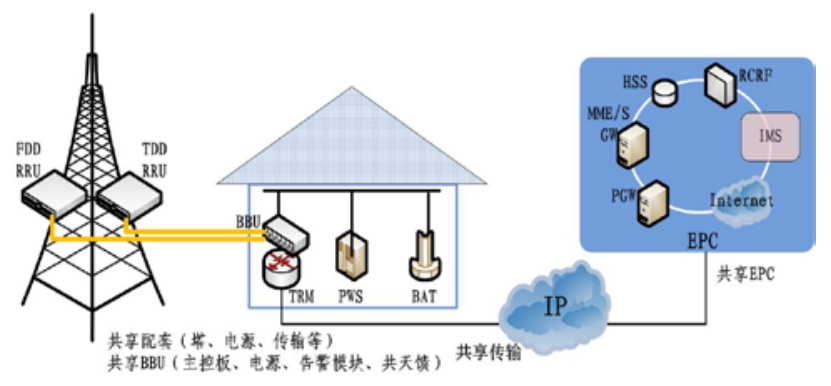

Fig. 1 Common mode networking technology

Common-mode networking has the following advantages:

(1) The design difference of frame structure between LTE TDD and LTE FDD resulted in system achieving differently. But their differences are mainly concentrated on the physical layer 
(PHY), and the difference between the radio link control layer (RLC) and the medium access control layer (MAC) is few. In high-level the design is almost the same. Statistics obtained only about $10 \%$ of the technical differences between LTE TDD and LTE FDD. This is very beneficial to achieve two systems at a lower cost, the interloper ability between LTE TDD and LTE FDD basically can be considered as the system interoperability.

(2) The shortage problem of FDD spectrum alleviated effectively. In the LTE network construction at the present stage, FDD standard was chose firstly in most network. But with the development of network construction, insufficient of spectrum resources and lack of capacity and other problems began to stand out. There are more spectrum resources in TDD. The combine with the FDD network will effectively alleviate the spectrum shortage.

(3) FDD and TDD common-mode Network take full advantages of both LTE FDD wide coverage and flexible technical characteristics of TDD spectrum allocation of resources and support for asymmetric traffic, giving users a better service experience.

\section{Relay technology.}

In order to deal with all kinds of complex wireless communication environment, to solve the problem of the actual network deployment, 3GPP in the LTE-Advanced (hereinafter referred to as LTE-A) R10 version of the protocol for the standardization of the relay. In the Acer station and the user terminal (UE) between a relay node, a direct link between the Acer station and the terminal is divided into two sections: the wireless link between Acer station and relay backhaul link (known as the Un interface on the return link function module corresponding to a relay node as transmission module, wireless link back) between the relay and terminal called access link (Uu interface, access link relay node corresponding to the $\mathrm{R}$ function module referred to access module). Through the reasonable deployment of the relay nodes, after the split of two links have a direct link than shorter transmission distance, and by reasonably setting can cause obstructions in spreading routes to further reduce, so after the split of two link compared with the radio propagation conditions of the direct link better.

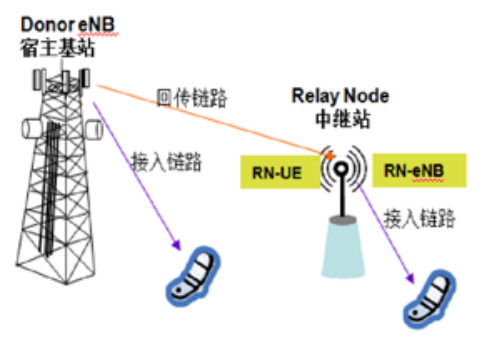

Fig.2 Relay technology Technical

The base station does not directly send the signal to the UE, first the signal is sent to a RN (Node Relay, relay node), and then sent to the UE by RN. This process is Relay technology. Relay technology can increase the system coverage, improve the link performance and cell throughput.

Relay base station equipment, it's just meet the wireless transmitting function, so it is simple to deploy, easy synchronization, convenient interference management; Relay base station equipment will not be using the traditional BBU+RRU architecture, but it integrates RF, baseband and antenna together, in order to meet the need of multi scene, all-weather LTE network construction.

Relay functional entities need to achieve the function which is mainly rendering eNB function in the Uu port, and in the Un port presents the function of UE. BBU Relay software structure see Fig.3. 


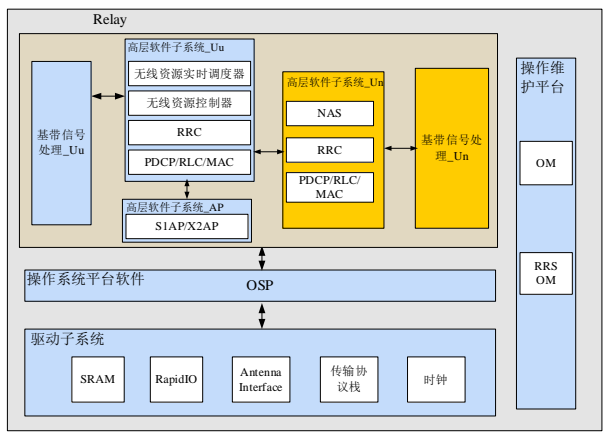

Fig.3 BBU software architecture of Relay

RN is logically divided into RN-UE (RUE) and RN-eNB (ReNB) two parts, RUE contains the standard UE part of the function and Relay enhancements, however, ReNB contains the standard eNodeB complete functions and Relay enhancements. The common UE access in the range of coverage by ReNB. At the same time, RUE access to the DeNB and set up LTE wireless backhaul for ReNB to provide air bearing. If the RN backhaul link and access links using the same frequency band, then known as the Internal work Relay band; if the RN backhaul link and access link using different carrier frequency, known as the external Relay band, The external Relay band can be as the first choice for most manufacturers, because the realization of a simple, easy to deploy.

\section{The power terminal of multi-mode multi frequency access system}

Some of important communication terminals which work in Power system transmission and distribution and other scenes are deployed in remote mountainous areas, basement or high-rise buildings and other public places of the scene, these signals may exist blind area coverage. In order to eliminate these blind spots, the scheme which is network coverage enhancement and multimode terminal public / private network fusion scheme can be used, only from the public point of view.

1) One scheme is the power terminal of multi-mode and multi frequency access system

The wireless network is used as access network, by using the special frequency. The wireless public network is the effective supplement to the wireless network, by using carrier frequency. The fusion scheme of public and private networks, can realize one aim of using the original public network coverage area, improving the capacity of the network by public network. Use multimode terminal to realize seamless switching between network and network security.

In case the 2/3/4G network mainly coexist in the current network, the power terminal generally access signal by choosing the best network, the terminal will switch along with the influence of various factors. Switching process: when the UE is in the connected state, it has three modes from E-UTRA to RAT: switch, CCO and redirect. All of these three methods are network controlled. Switch and CCO are UE in the transfer to connect E-UTRA to other RAT, that means to establish a connection or UE into other RAT. The difference between the two is that the switch is to make the UE connection from the connection state directly to the connection state, the user side data is still maintained transmission, while the CCO way is the first UE from the connection state into the idle (Idle), then make the connection state from the idle state into connected state, and the user plane data transmission will be interrupted. The redirection is make the E-UTRA in the connection state of the UE to the other RAT, and into the idle state. Switching and CCO can positioned to the target cell, while the redirection can only positioned to the target frequency point.

Only in the public area, the power terminal select public network access, activating the safety mechanism to ensure that data and information are security; only in electric power network in electric power network terminal access, data and information select transmission in network 
routing way; public network and electric power network are some regional security mechanism, the terminal starts priority access of electric power network in electric power network. The signal is weak, the terminal through the gravity access network, and activate the safety mechanism to ensure secure transmission of data and information.

2 ) Another scheme is the power terminal of multi-mode and multi frequency access system

LTE uses a flat network architecture, it reduces to 3 layers by deleting RNC nodes. Due to differences in standard and frequency bands, the LTE wireless network compared with the previous network, it presents "high bandwidth, small coverage" features, through heterogeneous network, this scheme can realize the high efficiency of power multi service, eliminate the blind area of power application, and realize the full use of network resources [3].

Macro / micro base station power terminal multi-mode multi frequency access system as shown in Fig. 4:

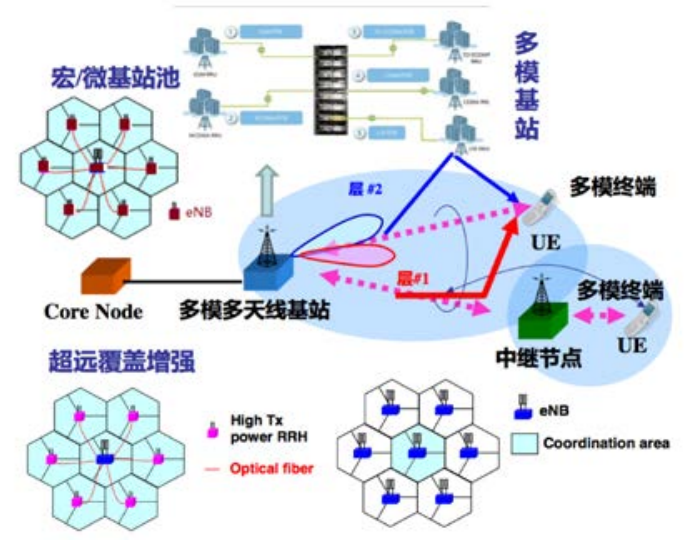

Fig. 4 Flexible access system structure diagram

As can be seen from Figure 4, the macro / micro base station power terminal multi-mode multi frequency access system includes:

TDD/FDD common mode access network, TDD/FDD common mode access network connect to the multi-mode base station wireless access network, TDD/FDD common mode access network at the same time to receive the TDD LTE and FDD LTE frame structure of the signal and transmission to the macro / micro base station pool;

The macro / micro base station connects to the multi-mode base station wireless access network, and the macro / micro base station pool is used to realize the wide coverage and weak coverage of the signal;

A multi-mode base station wireless access network is connected to a TDD/FDD common mode access network and a macro / micro base station pool, and a multi-mode base station wireless access network is used for integrated multi band, multi standard network.

Among them, the TDD/FDD common mode access network includes:

TDD RRU and TDD RRU set on the tower, TDD RRU connect to the BBU,

TDD RRU receives the signal frame of LTE TDD;

FDD RRU, FDD RRU set on the tower, FDD RRU connect to the BBU, FDD RRU is used to receive the signal frame of LTE FDD;

BBU, BBU connected to TRM, PWS and BAT, EPC connected to the shared BBU through TRM, BBU is used to receive RRU TDD and RRU FDD frame signal and sent to share EPC.

Among them, the macro / micro base pool includes:

A lot of macro base stations, Acer station is dispersed in space, used to achieve wide coverage;

A plurality of micro, micro base station, base station set in blind position formed in Acer station in order to achieve supplementary coverage.

Among them, the multi-mode base station wireless access network connect to the GSM network, WCDMA network, CDMA network, LTE network, that used to switch the signal between the GSM network, WCDMA network, and CDMA network. 


\section{Conclusion}

This paper presents a multi frequency power terminal access scheme based on macro and micro base station, Under the condition of the 2/3/4G network coexistence, the access scheme realizes by using of macro micro pool, TDD/FDD common network technology, wireless relay technology, the 2/3/4G network under the condition of coexistence, access to the power terminal. Multimode multi frequency power terminal transfer the data through a variety of heterogeneous network whenever and wherever possible, this scheme solves the access problems of mobile terminals such as electric power terminal access at transmission fixed position, mobile operation, mobile polling, and reduce the labor costs of the company communication network operation. Compared with the existing single wireless network service terminal, make full use of existing wireless network infrastructure, and limited spectrum resources ,multi-mode multi band terminal flexible access to network ,The access scheme reduces network operation complexity, saves maintenance cost, and improves the service level of the smart grid information communication.

\section{Acknowledgement}

In this paper, the research was sponsored by the Science and technology project of state Power Grid Corp (Project No. SGRIXTKJ[2015]349).

\section{Reference}

[1] Wu Hui min, Yang Ye.TD-LTE multi-mode multi band mobile communication terminal development.

[2] Wang Yong. Architecture of mobile communication base stations in the future - qualitative theory, method and practice, 2010

[3] IEEE Std C37. 118-2005 IEEE standard for synchro phasors for power systems[S]. New York, NY, USA: The Institute of Electrical and Electronics Engineers, 2006. 